\title{
Visualization of GFP-Expressing Tumors and Metastasis In Vivo
}

BioTechniques 30:1016-1026 (May 2001)

\author{
Robert M. Hoffman \\ AntiCancer, Inc., San Diego, \\ CA, USA
}

\section{INTRODUCTION}

A number of approaches have been taken to label tumor cells to visualize them in vivo. Previous attempts to genetically label tumor cells utilized the E. coli $\beta$-galactosidase (lacZ) gene to detect micrometastases $(25,26)$. How ever, lacZ detection requires extensive histological preparation with sacrifice of the tissue and/or animal; therefore, it was not possible to image, visualize, and study tumor cells in real time in viable fresh tissue or the live animal.

Fukumura et al. (16) and Chambers et al. (4) used videomicroscopy with a window placed over tumor tissue im planted in mice to visualize tumor tissue with fluorescent dyes. However, these methods are only suitable for a short time and cannot be used for longterm metastasis studies.

Weissleder et al. (36) have infused tumor-bearing animals with protease-activated, near-infrared fluorescent probes. Tumors with appropriate proteases could activate the probes and could be imaged externally. The limits to such a system include a much higher liver-totumor background ratio precluding imaging of liver metastasis, which is among the most important metastatic sites; the stated time limit of $96 \mathrm{~h}$ that precludes growth and efficacy studies; the requirement of appropriate tumor protease activity; and the requirement of selective tumor delivery of the probes.

Another attempt involved insertion of the luciferase gene into tumor cells such that they emit light (35). However, luciferase enzymes transferred to mam malian cells require the exogenous injected delivery of their luciferin sub- strate, an invasive and impractical requirement in an intact animal. The image resolution and signal of this approach is low, such that it takes minutes to acquire an image from an anesthetized animal (35). Also, it is not known whether luciferase genes can function stably over significant time periods in tumors and in the metastases derived from them.

It became clear that higher specificity, stronger signal, higher resolution, and physiological conditions are necessary to directly and externally optically image and follow the natural course or impediment of tumor progression and metastasis. The green fluorescent protein (GFP) gene, cloned from the bioluminescent jellyfish Aequorea victoria (31), was chosen to satisfy these conditions since it has demonstrated its great potential for use as a cellular marker $(3,5)$. GFP cDNA encodes a 283-amino acid monomeric polypeptide with a molecular weight of $27 \mathrm{kDa}(11,39)$ that requires no other Aequorea proteins, substrates, or cofactors to fluoresce (29). Recently, GFP gene gain-offunction bright mutants have been generated by various techniques (12$14,18)$ that have been humanized for high expression and signal (45).

We have developed technology that has enabled the stable transduction of the GFP gene into a large series of human tumor cell lines (6-10,38,40-43). The tumor cell lines have been selected to stably express GFP at high levels both in vitro and in vivo. We have demonstrated the important parameter that GFP-expressing cancer cells could be directly visualized in fresh tissues of transplanted animals, including in situ, 
at very high resolution down to the single-cell level (6-10). With this technology, we could visualize tumor cells that had seeded with or without subsequent colonization in all the major organs including liver, lung, brain, spinal cord, axial skeleton, and lymph nodes (6$10)$. Our recent results include the development of orthotopic GFP metastatic models of lung cancer (42), prostate cancer (40), melanoma (41), colon cancer (38), and pancreatic cancer (2). These results demonstrated that GFP gene-transfected tumor cells represent a powerful new tool to study tumor growth, angiogenesis, dormancy, dissemination, invasion, metastasis, and progression through all stages in vivo.

The high expression of GFP in the transplanted tumor cells has enabled the tumors in organs such as the colon, prostate, pancreas, brain, liver, lymph nodes, bone (38), and even the lung (B. Rashidi and R.M. Hoffman, manuscript in preparation) to be externally wholebody imaged by their fluorescence in the intact animals in a completely noninvasive manner.

\section{MATERIALS AND METHODS}

\section{Retroviral DNA Expression Vector}

The pLXIN retroviral vector pLEIN was purchased from Clontech Laboratories (Palo Alto, CA, USA). The pLEIN vector expresses enhanced green fluorescent protein (EGFP) and the neomycin resistance gene on the same bicistronic message that contains an internal ribosome entry site (IRES) $(40,42)$.

\section{Retroviral Production}

PT67, an NIH3T3-derived packaging cell line expressing the $10 \mathrm{Al}$ viral envelope, was purchased from Clontech Laboratories. PT67 cells were cultured in DMEM (Irvine Scientific, Santa Ana, CA, USA) supplemented with $10 \%$ heat-inactivated fetal bovine serum (FBS) (Gemini BioProducts, Calabasas, CA, USA). For vector production, PT67 packaging cells, at 70\% confluence, were incubated with a precipitated mixture of DOTAP ${ }^{\mathrm{TM}}$ reagent (Roche Molecular Biochemicals, Indianapolis, IN, USA), and saturating amounts of pLEIN plasmid for $18 \mathrm{~h}$. Fresh medium was replenished at this time. The cells were examined by fluorescence microscopy after $48 \mathrm{~h}$. For selection of GFP transductants, the cells were cultured in the presence of $500-2000 \mu \mathrm{g} / \mathrm{mL}$ of G418 (Life Technologies, Rockville, MD, USA) for seven days $(40,42)$.

\section{Retroviral Transduction of Tumor Cells and Selection of Stable High GFP Expression Clones}

For GFP gene transduction, 20\% confluent cancer cells were incubated with a $1: 1$ precipitated mixture of retroviral supernatants of PT67 cells and RPMI 1640 (Life Technologies) containing $10 \%$ FBS for $72 \mathrm{~h}$. Fresh medium was replenished at this time. Cells were harvested by trypsin/EDTA $72 \mathrm{~h}$ after infection and subcultured at a ratio of 1:15 into selective medium that contains $200 \mu \mathrm{g} / \mathrm{mL}$ G418. The level of G418 was increased stepwise to 800-1000 $\mu \mathrm{g} / \mathrm{mL}$. Stable GFP-expressing clones were then isolated in the absence of selective agent. Clones highly and stably expressing GFP were isolated with cloning cylinders (Bel-Art Products, Pequannock, NJ, USA) by trypsin/EDTA and were amplified and transferred by conventional culture methods $(40,42)$. After such selection procedures, the cells are highly stable, even after extensive passaging with all cells expressing GFP (Figure 1) $(30,38,40-43)$.

\section{Subcutaneous GFP Tumor Growth}

Nude mice were injected subcutaneously with a single dose of $10^{6}-10^{7}$ stable GFP tumor cell transductants. Cells were first harvested by trypsinization and washed three times with cold serum-containing medium, then kept on ice. Cells were injected in a total volume of $0.4 \mathrm{~mL}$ within $40 \mathrm{~min}$ of harvesting. Subcutaneously growing tumors were highly fluorescent (8) and occasionally gave rise to metastasis, which were readily visualized by GFP expression (41). The nude mice were sacrificed to harvest the tumor fragments three weeks after tumor cells injection $(8-10,40,42)$ for subsequent orthotopic implantation.

\section{Surgical Orthotopic Implantation of GFP Tumors}

Tumor fragments $\left(1 \mathrm{~mm}^{3}\right)$, stably expressing GFP, previously grown subcutaneously in nude mice, were implanted by surgical orthotopic implantation (SOI) on the appropriate organ in nude mice. After proper exposure of the organ to be implanted, 8-0 surgical sutures were used to penetrate the tumor pieces and attach them on the appropriate orthotopic organ. The incision in the skin was closed with a 7-0 surgical suture in one layer. The animals are kept under isoflurane anesthesia during surgery. All procedures of the operation described above are performed with a $7 \times$ magnification microscope (Leica ${ }^{\circledR}$ MZ6; Leica, Nussloch, Germany) (6$10,19,20,33,38,40-43)$.

\section{Experimental Metastasis of GFP Tumor Cells}

Nude mice were injected in the tail vein with a single dose of $10^{5}-10^{6}$ stable GFP-expressing cancer cells. Cells were first harvested by trypsinization and washed three times with cold serum-containing medium, then kept on ice. Cells were injected in a total volume of $0.8 \mathrm{~mL}$ serum-free medium within $40 \mathrm{~min}$ of harvesting. After various times, the mice were sacrificed, and fresh visceral organs and the skeleton were analyzed by fluorescence microscopy.

\section{Whole-Body Optical Imaging of GFP-Expressing Tumors}

Whole-body imaging apparatus for GFP (38). A Leica fluorescence stereo microscope, model LZ12, equipped with a $50-\mathrm{W}$ mercury lamp, was used for high-magnification imaging of GFP-expressing tumors and metastasis in situ or for whole-body imaging of animals with GFP-expressing tumors (38). Selective excitation of GFP was produced through a D425/60 bandpass filter and 470 DCXR dichroic mirror. Emitted fluorescence was collected through a longpass filter GG475 (Chroma Technology, Brattleboro, VT, USA) on a Hamamatsu C5810 3-chip cooled color CCD camera (Hamamatsu Photonics Systems, Bridgewater, NJ, 
USA). Images were processed for contrast and brightness and analyzed with the use of Image Pro Plus ${ }^{\mathrm{TM}} 3.1$ software (Media Cybernetics ${ }^{\circledR}$, Silver Springs, MD, USA). Images of $1024 \underline{x}$ 724 pixels were captured directly on an IBM PC or continuously through video output on a high-resolution Sony ${ }^{\circledR}$ VCR model SLV-R1000 (Sony, Tokyo, Japan). Whole-body imaging that visualized the entire animal at lower magnification was carried out in a light box illuminated by blue-light fiber optics (Lightools Research, Encinitas, CA, USA) and imaged using the thermoelectrically cooled color CCD camera, as described above.

\section{RESULTS}

\section{Ovarian Cancer}

GFP-expressing metastasis of orthotopic models of CHO-K1. Tumor fragments $\left(1 \mathrm{~mm}^{3}\right)$ of CHO-K1-GFP derived from the nude mouse subcutaneous GFP tumors were implanted by SOI on the ovarian serosa in nude mice $(8,43)$. All implanted mice had tumors in the ovaries. The tumor subsequently spread throughout the peritoneal cavity, including the colon, cecum, small intestine, spleen, and peritoneal wall. The primary tumor and peritoneal metastases were strongly fluorescent. $\mathrm{Nu}$ merous micrometastases were detected by fluorescence on the lungs of all mice. Multiple micrometastases were also detected by fluorescence on the liver, kidney, contralateral ovary, adrenal gland, para-aortic lymph node, and pleural membrane down to the singlecell level. Single-cell micrometastases could not be detected by standard histological techniques. Even multiple-cell small colonies were difficult to detect by hematoxylin and eosin staining, but they could be visualized clearly by GFP fluorescence $(8,43)$.

GFP-expressing experimental metastasis of CHO-K1. CHO-K1-GFP injected via the tail vein were visualized by GFP expression in the peritoneal wall vessels down to the single-cell level (8) (Figure 2). These cells formed emboli in the capillaries of the lung, liver, kidney, spleen, ovary, adrenal gland, thyroid gland, and brain (8).

\section{Lung Cancer}

GFP-expressing visceral metastasis of ANIP human lung cancer in orthotopic models. Tumor fragments (1 $\mathrm{mm}^{3}$ ) derived from the human lung cancer ANIP-GFP subcutaneous tumor growing in the nude mouse were implanted by SOI on the left lung in nude mice $(9,10)$. Patterns of contralateral and regional lung tumor metastases were visualized by GFP expression in orthotopic models $(9,10)$. Primary tumor grew in the implanted left lung in all mice after SOI of ANIP-GFP. The advancing margin of the tumor spreading in the ipsilateral lung was visualized by GFP expression. All animals explored had evidence of chest-wall invasion and local and regional spread.
Metastatic contralateral tumors involved the mediastinum, contralateral pleural cavity, and the contralateral visceral pleura. While the ipsilateral tumor had a continuous and advancing margin, the contralateral tumor seems to have been formed by multiple seeding events (Figure 3). Contralateral hilar lymph nodes were also involved as well as cervical lymph nodes visualized by GFP expression. When non-GFP-transfected ANIP was compared with GFPtransformed ANIP for metastatic capability similar results were seen $(9,10)$.

GFP-expressing bone metastases of H-460 human lung cancer in orthotopic models (42). Mice implanted by SOI with human lung cancer $\mathrm{H}-460$ GFP were sacrificed at three to four

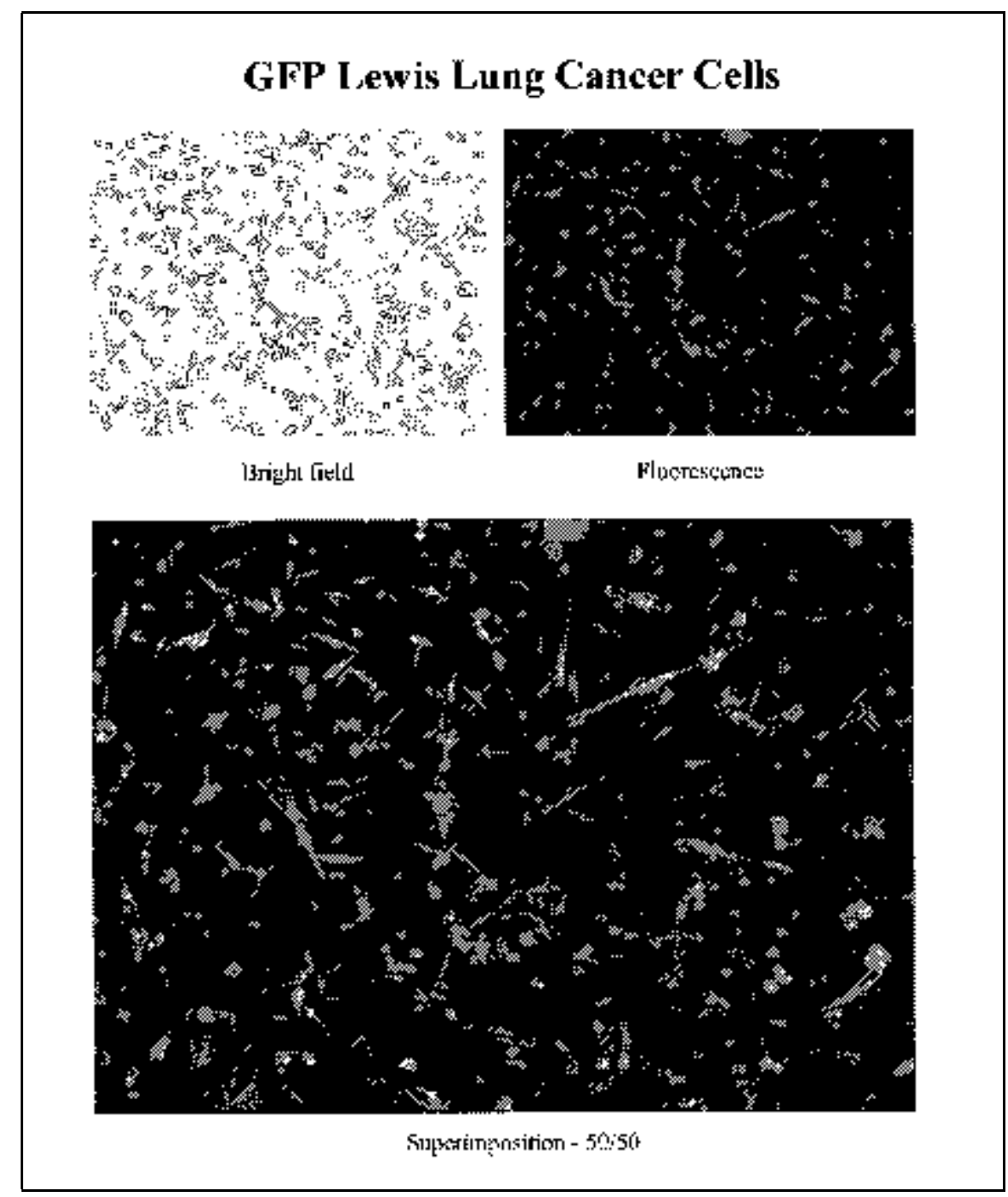

Figure 1. GFP-Lewis lung carcinoma cells shown under bright-field microscopy, fluorescence microscopy, and the two images superimposed. Superimposed images demonstrate all cells expressing GFP. 
weeks at the time of significant decline in performance status. GFP fluorescence demonstrated metastases in the left lung, the contralateral lung, chest wall, and the skeletal system. It was determined by GFP fluorescence that the vertebrae were the most involved skeletal sites of metastasis. Skull metastases were also visualized by GFP. Metastasis could also be visualized in the tibia and femur marrow by GFP fluorescence (42).

GFP-expressing experimental metastasis of ANIP human lung cancer. Human ANIP-GFP lung cancer cells were injected in the tail vein in nude mice that were sacrificed at four and eight weeks. In both groups, numerous micrometastatic colonies were detected in the whole lung tissue by GFP expression in fresh tissue (10). Even eight weeks after injection, most of the colonies were not obviously further developed compared with mice sacrificed at four weeks (10). Numerous small colonies, with 10 or less cells, were detected at the lung surface in both groups. Actively colonizing and dormant tumor cells were visualized in the lung (10). Dormant micrometastasis is one of the most important steps to understand in tumor progression (21). After eight weeks, metastases in the brain, the submandibular gland, lung, pancreas, bilateral adrenal glands, peritoneum, and pulmonary hilum lymph nodes were visualized by GFP expression (10).

\section{Prostate Cancer}

GFP-expressing visceral and bone metastasis of PC-3 human prostate cancer in orthotopic models. Two tumor fragments $\left(1 \mathrm{~mm}^{3}\right)$ from a high GFP-fluorescent subcutaneous tumor were implanted by SOI in the dorsolateral lobe of the prostate in nude mice (40). Skeletal metastases included the skull, rib, pelvis, femur, and tibia, visualized by GFP fluorescence. The tumors also metastasized to the lung, pleural membrane, kidney, liver, adrenal gland, brain, and spinal cord, all visualized by GFP fluorescence (40).

\section{Melanoma}

GFP-expressing visceral and bone metastasis of human and mouse melanoma. High GFP-expression malignant melanoma cell lines allowed the visualization of skeletal and multi-organ metastases after intravenous injection of B16-GFP cells in C57BL/6 mice and intradermal injection of LOX in nude mice (41). Extensive bone (Figure 4) and bone marrow metastases of B16F0 were visualized by GFP expression when the animals were sacrificed

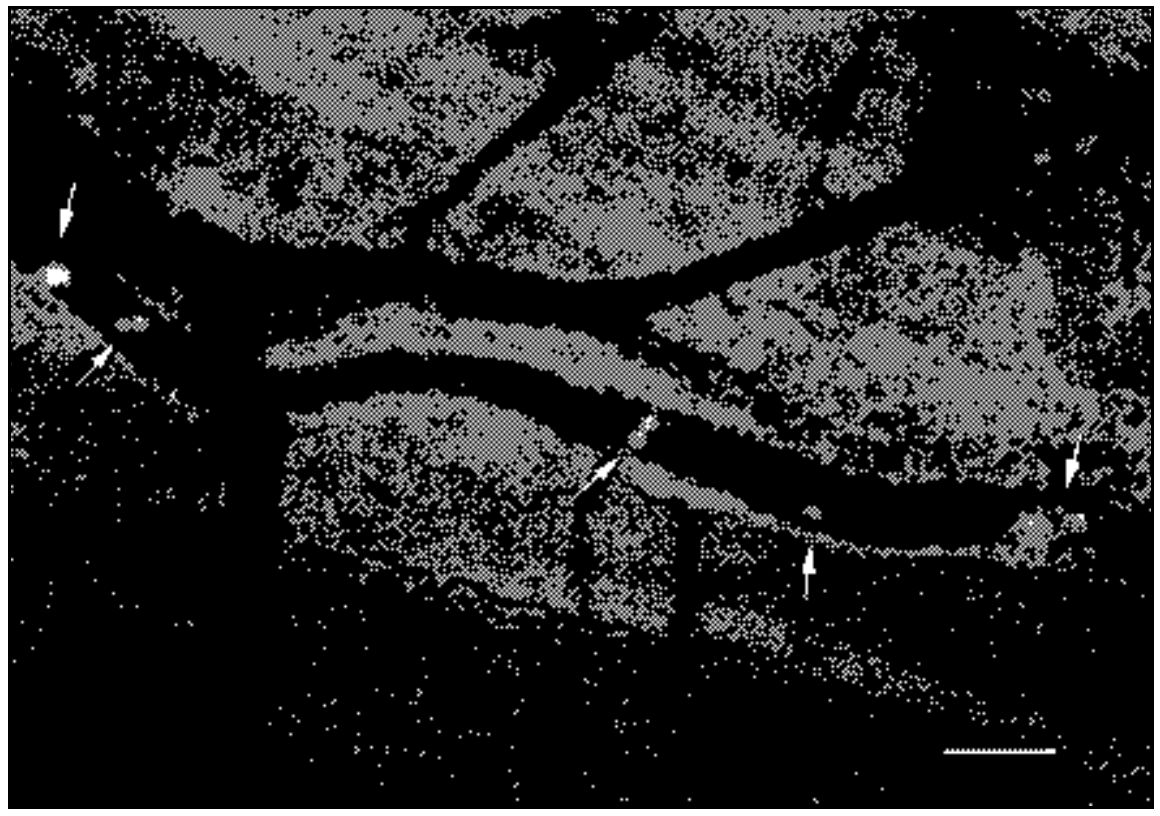

Figure 2. CHO-K1-GFP cells in a peritoneal vessel 2 min after tail-vein injection in nude mice (8). $\mathrm{Bar}=100 \mu \mathrm{m}$. 


\section{BioFeature}

Table 1. Metastatic Sites of GFP-Transduced Tumor Cell Lines

\begin{tabular}{|c|c|c|c|c|c|c|c|c|c|c|c|c|c|c|c|c|c|c|}
\hline \multirow{3}{*}{ 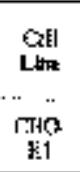 } & \multirow[b]{2}{*}{ Thiser } & \multirow[b]{2}{*}{$\begin{array}{l}\text { Typrar } \\
\text { ianded }\end{array}$} & \multirow[b]{2}{*}{ Eje } & \multirow[b]{2}{*}{ spicert } & \multirow[b]{2}{*}{$\operatorname{Line}$} & \multirow[b]{2}{*}{ Kath } & \multirow[b]{2}{*}{$\begin{array}{l}\text { lanish } \\
\text { :agki }\end{array}$} & \multirow[b]{2}{*}{ Ohark } & \multicolumn{3}{|c|}{ Melizotutb: Seles } & \multirow[b]{2}{*}{$\begin{array}{l}\text { : there } \\
\text { inte }\end{array}$} & \multirow[b]{2}{*}{ Stant } & \multirow[b]{2}{*}{ 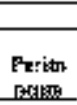 } & \multirow[b]{2}{*}{$\begin{array}{l}\text { Pars: } \\
\ldots x+\ldots+\ldots\end{array}$} & \multirow[b]{2}{*}{$\begin{array}{c}\text { Skr- } \\
\text { marken }\end{array}$} & \multirow[b]{2}{*}{ Braing } & \multirow[b]{2}{*}{ HE: } \\
\hline & & & & & & & & & 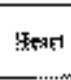 & $\begin{array}{l}\text { Adressa } \\
\text { Citanit. }\end{array}$ & 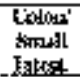 & & & & & & & \\
\hline & 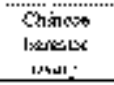 & bros & ' & r & - & + & + & $r$ & & 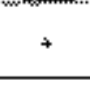 & 2 & & & \pm & & & & 8 \\
\hline \multirow[b]{2}{*}{ Ais } & \multirow{2}{*}{ 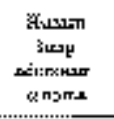 } & : MLv. & & & $:$ & \multicolumn{2}{|r|}{1.} & & & + & + & & & & & & & 9 \\
\hline & & 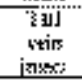 & & & + & & & & & & & & & & \multirow[t]{2}{*}{. } & & \multirow[t]{2}{*}{$=$} & \multirow[b]{2}{*}{$4 i$} \\
\hline$H-4 b)$ & 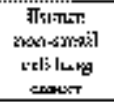 & 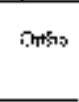 & & & + & & & & & & & & + & & & & & \\
\hline PC-: & 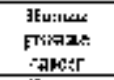 & Snall & + & & - & $=$ & & & & r & & & & & & & + & $4:$ \\
\hline \multirow{2}{*}{$\begin{array}{l}\text { Los } \\
\text { PAS }\end{array}$} & 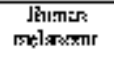 & \multirow{2}{*}{ 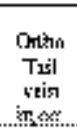 } & \multirow{2}{*}{+} & & \multirow[t]{2}{*}{ - } & \multirow[t]{2}{*}{$\div$} & + & & & + & & & \multicolumn{2}{|l|}{+} & & & + & 41 \\
\hline & 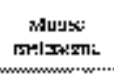 & & & & & & + & & & + & & & 1. & & & & 4 & $\$ 1$ \\
\hline KIL $x$ & 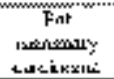 & Ortio & & & - & & + & & $\vdots$ & & & & & & & & & 3: \\
\hline 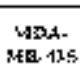 & 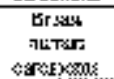 & 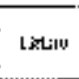 & & & - & & & & $\vdots$ & & & & & & & & & $\mathbf{w}$ \\
\hline 13,66 & $\begin{array}{l}\text { biat } \\
\text { Lastatary }\end{array}$ & . 나낭. & . $\ldots . .$. & 1 & - & & & & & & & + & & & & $\ldots$ & & It \\
\hline : & 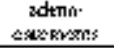 & $=c$. & + & & & & & & $\vdots$ & & & $\therefore$ & & & & & & \\
\hline 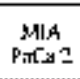 & 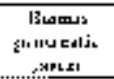 & (w) & + & + & & & ' & & & & & & & & & & & 2 \\
\hline $\mathrm{AnfE}-3$ & 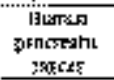 & L 财 ow & & + & & & + & & & & & & & $*$ & & & & $\ddot{z}$ \\
\hline 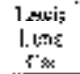 & 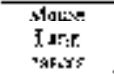 & Datas & & & - & & + & & $\div$ & & & & & & & - & + & בx \\
\hline
\end{tabular}

three weeks after cell implantation. For both cell lines metastases were visualized in many organs, including brain, lung, pleural membrane, liver, kidney, adrenal gland, lymph nodes, skeleton, muscle, and skin by GFP fluorescence. This was the first observation of experimental skeletal metastases of melanoma and made possible by GFP expression.

\section{Pancreatic Cancer}

GFP-expressing metastasis of MIA-PaCa2-GFP and BxPC-3-GFP human pancreatic cancer in orthotopic models (2). MIA-PaCa2-GFP and BxPC-3-GFP tumor fragments were transplanted by SOI to the nude mouse pancreas for fluorescence visualization of the chronology of pancreatic tumor growth and metastatic targeting. BxPC-3-GFP tumors developed rapidly in the pancreas and spread regionally to the spleen and retroperitoneum as early as six weeks. Distant metastases in BxPC-3-GFP were rare.
In contrast, MIA-PaCa-2-GFP grew more slowly in the pancreas but rapidly metastasized to distant sites including liver and portal lymph nodes. Regional metastases in MIA-PaCa-2-GFP were

rare. These studies demonstrate that pancreatic cancers have highly specific and individual "seed-soil" interactions governing the chronology and sites of metastatic targeting (2).

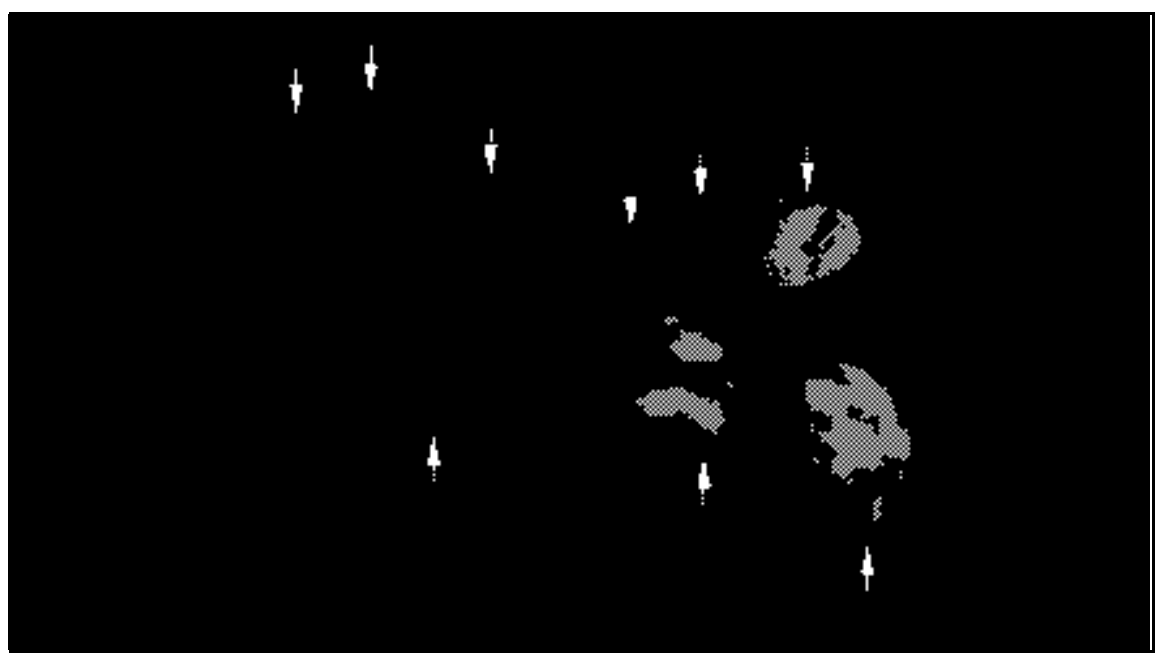

Figure 3. Metastasis of human lung cancer ANIP-GFP occurring in the contralateral right lung after ANIP-GFP tumor tissue was surgically orthotopically implanted in the left lung of nude mice. At sacrifice and removal of the contralateral lung, both large metastatic colonies and small metastatic seeds were observed in the contralateral lung by GFP expression (arrowheads) (19). 


\section{BioFeature}

\section{Brain Cancer}

GFP-expressing tumor growth of Daoy human medulloblastoma in orthotopic models (27). Stable GFP-expressing meduloblastoma clones were isolated and stereotactically injected into the frontal cortex of nude mice. Four weeks later, whole brain sections were examined. GFP-transduced Daoy cells maintained stable high-level GFP expression in the brain. GFP fluorescence visualized the primary tumor margins. Locally invasive foci, including those immediately adjacent to the tumor's leading invasive edge, were visualized only by GFP fluorescence and could not be seen by standard histological techniques (27).

\section{Breast Cancer}

GFP-expressing metastasis of MDA-MB-435-GFP human breast cancer in orthotopic models (34). Flow cytometry was used to characterize GFP-expressing breast tumor cells that had metastasized to the lung from the tumor implanted orthotopically in the mammary fat pad. In vivo selection was used to isolate a variant with increased primary tumor growth and metastasis. Metastatic cells migrated to the lungs of SCID mice very early after orthotopic implantation and increased continuously until death approximately eight weeks later. One GFP-fluorescent tumor cell per 200000 host cells could be detected in dissociated tissues by flow cytometry. Levels of circulating tumor cells in blood were also visualized by GFP at an early timepoint (34).

Visualization of motility by GFP-

Figure 4. Bone metastasis of B16F0-GFP (41). (a) Skull, top: no metastasis was detected under bright field microscopy. Bar $=640 \mu \mathrm{m}$. Bottom: shows same area as top part, bone metastasis visualized in the skull under fluorescent microscopy (b) Vertebral body, top: no metastasis was detected under bright-field microscopy. $\mathrm{Bar}=1280 \mu \mathrm{m}$. Bottom shows same area as top part, bone metastasis visualized in the vertebral body under fluorescent microscopy. (c) Bone metastases visualized by GFP expression in humerus and scapula. $\mathrm{Bar}=1280 \mu \mathrm{m}$. (d) Bone metastases visualized by GFP expression in the distal end of the femur. Bar $=1280 \mu \mathrm{m}$. (e) Bone metastases visualized by GFP expression in the head of the femur. Bar = $800 \mu \mathrm{m}$. (f) Bone metastases visualized by GFP expression in pelvis. $\mathrm{Bar}=1280 \mu \mathrm{m}$. expressing in a rat breast tumor in orthotopic models (15). A metastatic rat breast cancer cell line was established that constitutively expresses GFP. After injection of these cells into the mammary fat pad of female Fischer 344 rats, primary and metastatic tumors were visualized by GFP expression after removal of the skin. Intravital imaging of the GFP-expressing primary tumor in situ with a laser-scanning confocal microscope visualizing the motility patterns of the tumor cells (15).

Metastatic behavior of the GFP-expressing tumors described above is summarized in Table 1.

\section{GFP Tumor Cells in Blood Vessels}

Single tumor cells could be seen in blood vessels after tail-vein injection of
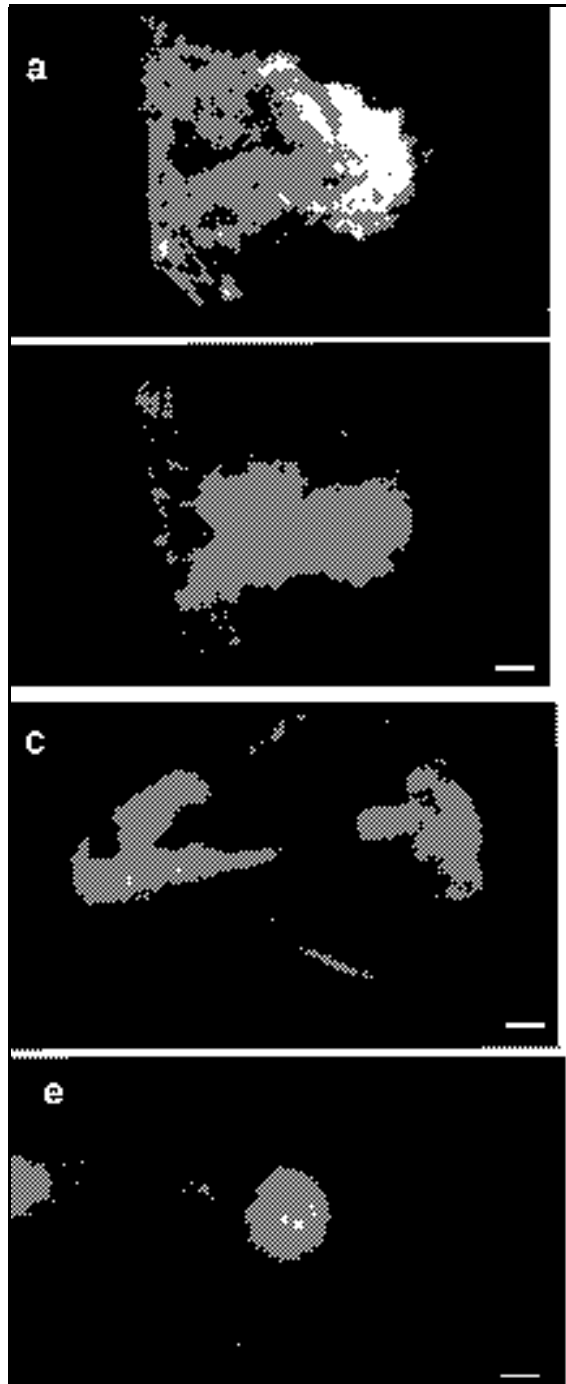

tumor cells stably expressing GFP (8). Naumov et al. (30), using intravital microscopy, visualized GFP tumor cells colonizing various organs after extravasation. Huang et al. (22) and Li et al. (24) visualized tumor cell-vessel interaction by GFP in skin window chambers in rodents and observed angiogenic effects very early in tumor colony formation. Wyckoff et al. (37) in an orthotopic mammary-pad injection model also visualized tumor-vessel interaction by GFP expression of the tumor cells. Al-Mehdi et al. (1) visualized what they claimed to be intravascular tumor colony formation by GFP expression in a lung perfusion study. Moore et al. (28) visualized vessels in a GFP-expressing rodent cell line.

Intravital videomicroscopy of GFP-expressing tumors. Previously,
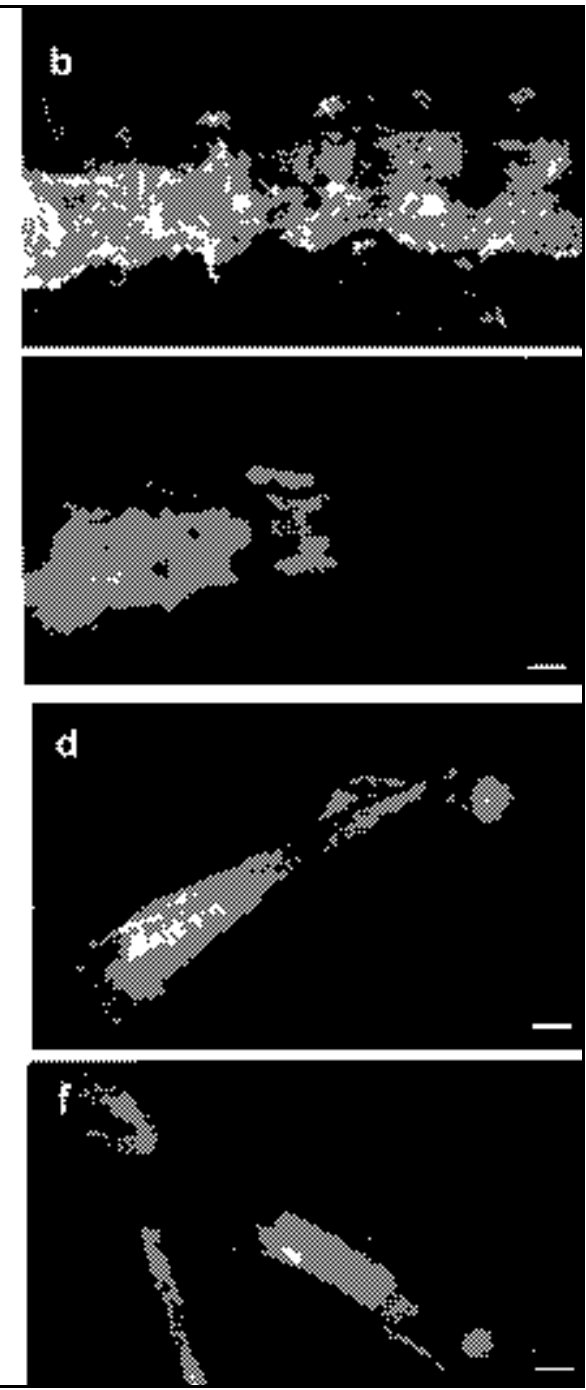
cells have been visualized in vivo using exogenously added fluorescent labels, limiting observations to a few cell divisions, or by natural markers (e.g., melanin) expressed only by specific cell types. Using $\mathrm{CHO}-\mathrm{K} 1$ cells that stably express GFP, intravital videomicroscopy visualized sequential steps in metastasis within mouse liver, from initial arrest of cells in the microvasculature to the growth and angiogenesis of metastases (30). Individual, non-dividing cells, as well as micro- and macro-metastases, were visualized and quantified, including cellular details such as pseudopodial projections. GFP visualization demonstrated preferential growth and survival of micrometastases near the liver surface. A small population of single cells that persisted over the 11-day observation period was visualized, which may represent dormant tumor cells (30).

Whole-body fluorescence optical tumor imaging of tumor growth and metastasis (38). Whole-body optical images visualized metastatic lesions of GFP-expressing tumors in the colon, prostate, brain, liver (Figure 5), pancreas, lymph nodes, and bone in transplanted mice. These images were used for real-time, quantitative measurement of primary and metastatic tumor growth in each of these organs. The depth to which metastasis and micrometastasis could be imaged depended on their size. The simple, noninvasive, and highly selective imaging technology, made possible by the strong GFP fluorescence, enabled the detailed visualization of tumor growth and metastasis formation. Optical imaging based on fluorescence expression of GFP can solve critical problems of other methods of imaging. The most important is that it can allow for a high ratio of the fluorescence signal from the tumor compared to the autofluorescence from any background tissue. It does not require other molecules such as monoclonal antibodies or luciferin or other substrates to target the tumor and metastases for imaging. It does not require tumor-specific proteases or delivery of fluorescent probes. It also does not require the use of harmful ionizing radiation and contrast agents used in standard imaging techniques. GFPbased optical imaging can also take advantage of very sensitive, thermoelec- trically cooled CCD cameras to acquire the images. The GFP signal is so strong and selective that external images of GFP-expressing tumors and their metastases can be acquired in freely moving animals. This new GFP imaging technology will facilitate in vivo high-throughput screening of antitumor and antimetastatic drugs (38).

Selective in vivo tumor delivery of the GFP gene to report future occurrence of metastasis. The GFP gene was administered to intraperitoneally growing human stomach cancer in nude mice to visualize future regional and distant metastases (17). GFP retroviral supernatants were injected i.p. from day-4 to day-10, following i.p. implantation of the NUGC-4 human stomach cancer cells. Tumor and metastasis fluorescence was visualized every other week with the use of fluorescence optics (described above) via a laparotomy on the tumor-bearing animals. No normal tissues were found to be transduced by the GFP retrovirus. Two weeks after retroviral GFP delivery, GFP-expressing tumor cells were observed in gonadal fat, greater omentum, and intestine, indicating that these primary intraperitoneally growing tumors were efficiently transduced by the GFP gene and could be visualized by its expression. At the second and third laparotomies at four and six weeks, respectively, GFP-expressing tumor cells were found spreading to lymph nodes in the mesentery. At the fourth laparotomy at eight weeks, widespread tumor growth including liver metastasis was observed. Thus, reporter-gene transduction of the primary tumor enabled detection of its subsequent metastasis. This reporter-gene therapy model could be applied to primary tumors before resection or other treatment to have a fluorescent early detection system for metastasis and recurrence (17).

Recently, GFP on an HSV-1/EBV vector was administered to tumor-bearing animals. However, long-time GFP expression did not seem to be achieved (32).

\section{CONCLUSIONS}

Tumor cells stably expressing GFP in vivo are a powerful new tool for cancer research. With regard to stability, Naumov et al. (30) noted that all the
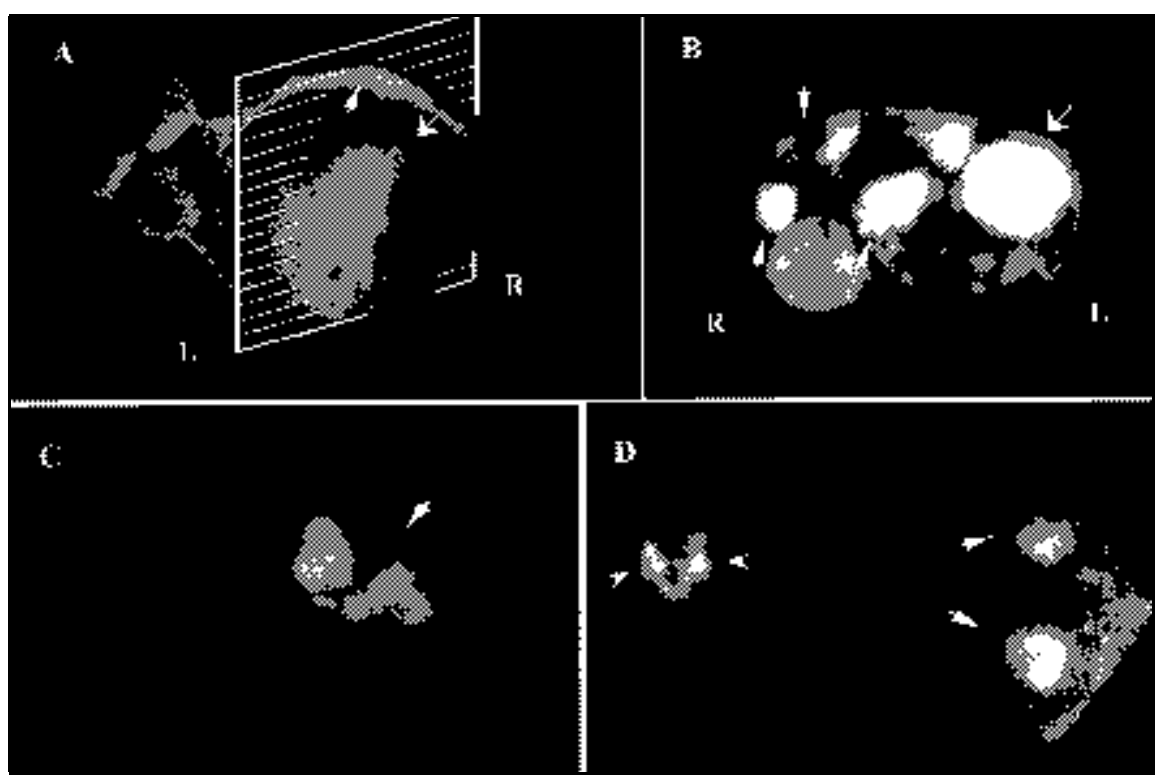

Figure 5. External and internal images of liver metastases (38). (a) Lateral, whole-body image of metastatic liver lesions of a GFP-expressing human colon cancer in the left and right lobes of a live nude mouse at day 21 after SOI. (b) Physical cross-section of mouse shown in Figure 5a corresponding to the level of the external image of the tumor in the liver that was acquired. Fine arrows show metastatic lesions in the right lobe of liver, and the thick arrows show the metastatic lesion in the left lobe of the liver. (c) Fluorescent whole-body ventral image of the primary colon tumor. (d) Dorsal external image of metastatic tumor in the caudal region of the left and right lobes of the liver (thick arrows) and skull metastasis (arrowheads). 
CHO-K1-GFP cells used in their study exhibited stable fluorescence as demonstrated by flow cytometry, even after 24 days of maintenance in vitro in medium deprived of selective pressure. This high stability suggested stability of GFP expression of the cells in vivo, which seems to be the case for all tumor types thus far studied because they generate extensive GFP-expressing metastases (Table 1).

When the GFP-expressing tumor cells were transplanted in mice, their visualization in fresh tissue, or the live animal, down to the single-cell level $(8,30)$ gave new insight into the realtime growth and metastatic behavior of cancer. See Table 1 for a summary of the metastatic behavior of GFP tumor cells in orthotopic and other animal models. A number of independent studies $(8,10,15)$ have demonstrated that GFP transduction and expression does not affect metastatic behavior, including an extensive comparison of metas- tases of a GFP-transduced rat mammary carcinoma and its parental line (15).

GFP as a cytoplasmic marker (which can be transfected into any cell type of interest) enabled the visualization in vivo of both the general outlines of cells and fine morphological details such as long slender pseudopodial projections, for extended periods of time (30).

In very important developments, stable high GFP expression of tumor cells has enabled the whole-body imaging of tumor and metastastic growth in a com pletely noninvasive manner (38). For the first time, tumor growth and metastatic studies, including drug evaluations, can be performed and quantified in real-time in single animals. The potential of this technology is very high.

A further advantage of GFP-expressing cells is the increased contrast between brightly fluorescent tumor tissue and blood vessels within it. The ability to visualize and quantify blood vessel development in metastases in vivo will greatly facilitate studies of angiogenesis and the testing of effects of anti-angiogenic agents on metastatic development (44).

Much important new information and drug discovery should soon follow with the use of GFP tumor cells in vivo.

\section{REFERENCES}

1.Al-Mehdi, A.B., K. Tozawa, A.B. Fisher, L. Shientag, A. Lee, and R.J. Muschel. 2000. Intravascular origin of metastasis from the proliferation of endothelium-attached tumor cells: a new model for metastasis. Nat. Med. 6:100102.

2.Bouvet, M., M. Yang, S. Nardin, X. Wang, P. Jiang, E. Baranov, A.R. Moossa, and R.M. Hoffman. 2000. Chronologically-specific metastatic targeting of human pancreatic tumors in orthotopic models. Clin. Exp. Metastasis 18:213-218.

3.Chalfie, M., Y. Tu, G. Euskirchen, W.W. Ward, and D.C. Prasher. 1994. Green fluorescent protein as a marker for gene expression. Science 263:802-805.

4.Chambers, A.F., I.C. MacDonald, E.E. 
Schmidt, S. Koop, V.L. Morris, R. Khokha, and A.C. Groom. 1995. Step in tumor metastasis: new concepts from intravital videomicroscopy. Cancer Metastasis Rev. 14:279-301.

5.Cheng, L., J. Fu, A. Tsukamoto, and R.G. Hawley. 1996. Use of green fluorescent protein variants to monitor gene transfer and expression in mammalian cells. Nat. Biotechnol. 14:606-609.

6.Chishima, T., M. Yang, Y. Miyagi, L. Li, Y. Tan, E. Baranov, H. Shimada, A.R. Moossa, S. Penman, and R.M. Hoffman. 1997. Governing step of metastasis visualized in vitro. PNAS 94:11573-11576.

7.Chishima, T., Y. Miyagi, L. Li, Y. Tan, E. Baranov, M. Yang, H. Shimada, A.R. Moossa, and R.M. Hoffman. 1997. The use of histoculture and green fluorescent protein to visualize tumor cell host interaction. In Vitro Cell. Dev. Biol. Anim. 33:745-747.

8.Chishima, T., Y. Miyagi, X. Wang, H. Yamaoka, H. Shimada, A.R. Moossa, and R.M. Hoffman. 1997. Cancer invasion and micrometastasis visualized in live tissue by green fluorescent protein expression. Cancer Res. 57:2042-2047.

9.Chishima, T., Y. Miyagi, X. Wang, E. Baranov, M. Yang, Y. Tan, H. Shimada, A.R. Moossa, and R.M. Hoffman. 1997. Metastatic patterns of lung cancer visualized live and in process by green fluorescent protein expression. Clin. Exp. Metastasis 15:547-552.

10.Chishima, T., Y. Miyagi, X. Wang, Y. Tan, H. Shimada, A.R. Moossa, and R.M. Hoffman. 1997. Visualization of the metastatic process by green fluorescent protein expression. Anticancer Res. 17:2377-2384.

11.Cody, C.W., D.C. Prasher, W.M. Westler, F.G. Prendergast, and W.W. Ward. 1993. Chemical structure of the hexapeptide chromophore of the Aequorea green fluorescent protein. Biochemistry 32:1212-1218.

12. Cormack, B., R. Valdivia, and S. Falkow. 1996. FACS-optimized mutants of the green fluorescent protein (GFP). Gene 173:33-38

13.Crameri, A., E.A. Whitehorn, E. Tate, and W.P.C. Stemmer. 1996. Improved green fluorescent protein by molecular evolution using DNA shuffling. Nat. Biotechnol. 14:315-319.

14.Delagrave, S., R.E. Hawtin, C.M. Silva, M.M. Yang, and D.C. Youvan. 1995. Redshifted excitation mutants of the green fluorescent protein. Bio/Technology 13:151-154.

15.Farina, K.L., J.B. Wyckoff, J. Rivera, H. Lee, J.E. Segall, J.S. Condeelis, and J.G. Jones. 1998. Cell motility of tumor cells visualized in living intact primary tumors using green fluorescent protein. Cancer Res. 58:2528-2532.

16.Fukumura, D., F. Yuan, W.L. Monsky, Y Chen, and R.K. Jain. 1997. Effect of host microenvironment on the microcirculation of human colon adenocacinoma. Am. J. Pathol. 151:679-688.

17.Hasegawa, S., M. Yang, T. Chishima, H. Shimada, A.R. Moossa, and R.M. Hoffman. 2000. In vivo tumor delivery of the green fluorescent protein gene to report future occurrence of metastasis. Cancer Gene Ther. 7:1336-1340.

18.Heim, R., A.B. Cubitt, and R.Y. Tsien. 1995 Improved green fluorescence. Nature 373:663664.
19.Hoffman, R.M. 1999. Green fluorescent protein to visualize cancer progression and metastasis, p. 20-31. In P. Michael Conn (Ed.), Methods in Enzymology, Green Fluorescent Protein, Vol. 302. Academic Press, San Diego.

20.Hoffman, R.M. 1999. Orthotopic metastatic mouse model for anticancer drug discovery evaluation: a bridge to the clinic. Invest. New Drugs 17:343-359.

21.Holmgren, L., M.S. O'Reilly, and J. Folkman. 1995. Dormancy of micrometastases: balanced proliferation and apoptosis in the presence of angiogenesis suppression. Nat. Med. 1:149-153.

22.Huang, Q., S. Shan, R.D. Braun, J. Lanzen, G. Anyrhambatla, G. Kong, M. Borelli, P. Corry, M.W. Dewhirst, and C.Y. Li. 1999. Noninvasive visualization of tumors in rodent dorsal skin window chambers. Nat. Biotechnol. 17:1033-1035.

23.Kan, Z. and T-J. Liu. 1999. Video microscopy of tumor metastasis; using the green fluorescent protein (GFP) gene as a cancer-cell-labeling system. Clin. Exp. Metastasis 17:49-55

24.Li, C.Y., S. Shan, Q. Huang, R.D. Braun, J. Lanzen, K. Hu, P. Lin, and M.W. Dewhirst. 2000. Initial stages of tumor cell-induced angiogenesis: evaluation via skin window cham bers in rodent models. J. Natl. Cancer Inst. 92:143-147.

25.Lin, W.C. and L.A. Culp. 1992. Altered establishment/clearance mechanisms during experimental micrometastasis with live and/or disabled bacterial lac-tagged tumor cells. Invasion Metastasis 12:197-209.

26.Lin, W.C., T.P. Pretlow, T.G. Pretlow, II, and L.A. Culp. 1990. Bacterial lacZ gene as a highly sensitive marker to detect micrometastasis formation during tumor progression. Cancer Res. 50:2808-2817.

27.MacDonald, T.J., P. Tabrizi, H. Shimada B.V. Zlokov, and W.E. Laug. 1998. Detection of brain tumor invasion and micrometastasis in vivo by expression of enhanced green fluorescent protein. Neurosurgery 43:1437-1442.

28.Moore, A., E. Marecos, M. Simonova, R. Weissleder, and A. Bogdanov, Jr. 1998. Novel gliosarcoma cell line expressing green fluorescent protein: a model for quantitative assessment of angiogenesis. Microvasc. Res. 56:145153.

29.Morin, J. and J. Hastings. 1971. Energy transfer in a bioluminescent system. J. Cell Physiol. 77:313-318.

30.Naumov, G.N., S.M. Wilson, I.C. MacDonald, E.E. Schmidt, V.L. Morris, A.C. Groom, R.M. Hoffman, and A.F. Chambers. 1999. Cellular expression of green fluorescent protein, coupled with high-resolution in vivo videomicroscopy, to monitor steps in tumor metastasis. J. Cell Sci. 112:1835-1842.

31.Prasher, D.C., V.K. Eckenrode, W.W. Ward, F.G. Prendergast, and M.J. Cormier. 1992. Primary structure of the Aequorea victoria green-fluorescent protein. Gene 111:229-233.

32.Qi, J., C.J. Link, and S. Wang. 2000. Direct observation of GFP gene expression transduced with HSV-1/EBV amplicon vector in unfixed tumor tissue. BioTechniques 28:206-208.

33. Rashidi, B., M. Yang, P. Jiang, E. Baranov, Z. An, X. Wang, A.R. Moossa, and R.M Hoffman. 2000. A highly metastatic Lewis lung carcinoma orthotopic green fluorescent protein model. Clin. Exp. Metastasis 18:57-60.

34.Schmidt, C.M., S.L. Settle, J.L. Keene, W.F. Westlin, G.A. Nickols, and D.W. Griggs. 1999. Characterization of spontaneous metastasis in an aggressive breast carcinoma model using flow cytometry. Clin. Exp. Metastasis 17:537-544.

35.Sweeney, T.J., V. Mailander, A.A. Tucker, A.B. Olomu, W. Zhang, Y. Cao, R.S. Negrin, and C.H. Contag. 1999. Visualizing the kinetics of tumor-cell clearance in living animals. Proc. Natl. Acad. Sci. USA 96:12044-12049.

36. Weissleder, R., C.H. Tung, U. Mahmood, and A. Bogdanov, Jr. 1999. In vivo imaging of tumors with protease-activated near-infrared fluorescent probes. Nat. Biotechnol. 17:375-378.

37.Wyckoff, J.B., J.G. Jones, J.S. Condeelis, and J.E. Segall. 2000. A critical step in metastasis: in vivo analysis of intravasation at the primary tumor. Cancer Res. 60:2504-2511.

38. Yang, M., E. Baranov, P. Jiang, F-X. Sun, XM. Li, L. Li, S. Hasegawa, M. Bouvet et al. 2000. Whole-body optical imaging of green fluorescent protein-expressing tumors and metastases. Proc. Natl. Acad. Sci. USA 97:1206-1211.

39.Yang, F., L.G. Miss, and G.N. Phillips, Jr. 1996. The molecular structure of green fluorescent protein. Nat. Biotechnol. 14:1246-1251.

40. Yang, M., P. Jiang, F.X. Sun, S. Hasegawa, E. Baranov, T. Chishima, H. Shimada, A.R. Moossa, and R.M. Hoffman. 1999. A fluorescent orthotopic bone metastasis model of human prostate cancer. Cancer Res. 59:781-786.

41. Yang, M., P. Jiang, Z. An, E. Baranov, L. Li, S. Hasegawa, M. Al-Tuwaijri, T. Chishima, H. Shimada, A.R. Moossa, and R.M. Hoffman. 1999. Genetically fluorescent melanoma bone and organ metastasis models. Clin. Cancer Res. 5:3549-3559.

42. Yang, M., S. Hasegawa, P. Jiang, X. Wang, Y. Tan, T. Chishima, H. Shimada, A.R. Moossa, and R.M. Hoffman. 1998. Widespread skeletal metastatic potential of human lung cancer revealed by green fluorescent protein expression. Cancer Res. 58:4217-4221.

43. Yang, M., T. Chishima, X. Wang, E. Baranov, H. Shimada, A.R. Moossa, and R.M. Hoffman. 1999. Multi-organ metastatic capability of Chinese hamster ovary cells revealed by green fluorescent protein (GFP) expression. Clin. Exp. Metastasis 17:417-422.

44. Yang, M., E. Baranov, X-M. Li., J-W. Wang, P. Jiang, L. Li, A.R. Moossa, S. Penman, and R.M. Hoffman. 2001. Whole-body and intravital optical imaging of angiogenesis in orthotopically implanted tumors. Proc. Natl. Acad. Sci. USA 98:2616-2621.

45.Zolotukhin, S., M. Potter, W.W. Hauswirth, J. Guy, and N. Muzycka. 1996. "Humanized" green fluorescent protein cDNA adapted for high-level expression in mammalian cells. J. Virol. 70:4646-4654.

Address correspondence to:

Robert M. Hoffman, Ph.D.

AntiCancer, Inc.

7917 Ostrow Street

San Diego, CA 92111, USA

e-mail: all@anticancer.com 